\title{
Stabilization of the $T$ system by an integrable deformation
}

\author{
Cristian Lăzureanu ${ }^{1, *}$ and Cristiana Căplescu ${ }^{1, * *}$ \\ ${ }^{1}$ Politehnica University of Timişoara, P-ta Victoriei 2, 300 006, Timişoara, ROMANIA
}

\begin{abstract}
In this paper, some deformations of the $T$ system are constructed. In order to stabilize the chaotic behavior of the $T$ system, we particularize these deformations obtaining some external linear control inputs. In each case, we prove that the controlled system is asymptotically stable.
\end{abstract}

\section{Introduction}

The nonlinear phenomena that appear in many fields, such as engineering, physics, chemistry, economics, and biology, may be modeled by nonlinear systems [1, 4, 18, 19, 24]. On the one hand, in some situations, it is useful that such dynamics to be chaotic. Thus "for a given system, which may be linear or nonlinear and originally can be nonchaotic or even stable, the aim is to create chaos by using a simple and implementable controller (e.g. a parameter turner or a state feedback controller)" [17]. On the other hand chaos may be harmful. In such cases, some controllers are used to suppress the chaotic dynamical behavior $[2,4,6]$.

In this paper, we use some linear control inputs to stabilize the chaotic dynamical behavior of the $T$ system [21, 23]. Mention that we use other functions than those used in the papers [5] and [22]. In fact, we obtain the control functions using some deformations of the $T$ system, which are given by the integrable deformations method considered in [10], method also used in $[13,15]$. For the Euler top, the alteration of its constants of motion leads to some integrable deformations [7]. Moreover, integrable deformations of a three-dimensional Hamilton-Poisson system is a new Hamilton-Poisson system, which can be viewed as a controlled system $[8,9,11,12,14]$.

The paper is organized as follows. In Section 2, we briefly recall the integrable deformations method for a three-dimensional system of differential equations [10], and we construct some integrable deformations of the $T$ system. In Section 3, we particularize these deformations. We obtain linear feedback controls, and we prove that the controlled system is asymptotically stable in each case. We study the stabilization of the $T$ system via linear controls about one, two, and three axes, respectively.

\section{Some deformations of the $T$ system}

In this section we give some deformations of the $T$ system (2). We use the integrable deformations method for a three-dimensional system of differential equations [10]. Depending on

\footnotetext{
*e-mail: cristian.lazureanu@upt.ro

**e-mail: cristiana.caplescu@upt.ro
} 
the considered Hamilton-Poisson part of the system $T$, many families of integrable deformations can be obtained. A family of such deformations was obtained in [15]. In the following we give another family of integrable deformations of the $T$ system.

For a system of the form $\dot{\mathbf{x}}=\mathbf{f}, \mathbf{f}=\left(f_{1}, f_{2}, f_{3}\right)$ we write $\mathbf{f}(\mathbf{x})=\mathbf{g}(\mathbf{x})+\mathbf{h}(\mathbf{x})$ such that the system $\dot{\mathbf{x}}=\mathbf{g}(\mathbf{x})$ is a Hamilton-Poisson system, that is it has two functionally independent constants of motion, $H=H(\mathbf{x})$ and $C=C(\mathbf{x})$. In addition, this Hamilton-Poisson system can be written in the form $\dot{\mathbf{x}}=\mu \nabla H \times \nabla C$, where $\mu \in C^{1}$ on an open set $\Omega \subseteq \mathbb{R}^{3}$.

In this framework, an integrable deformation of the system $\dot{\mathbf{x}}=\left(f_{1}, f_{2}, f_{3}\right)$ is given in [10] by

$$
\left\{\begin{array}{rl}
\dot{x} & =f_{1}(x, y, z)+\mu\left(H_{y} \beta_{z}-H_{z} \beta_{y}+\alpha_{y} C_{z}-\alpha_{z} C_{y}+\alpha_{y} \beta_{z}-\alpha_{z} \beta_{y}\right) \\
\dot{y} & =f_{2}(x, y, z)-\mu\left(H_{x} \beta_{z}-H_{z} \beta_{x}+\alpha_{x} C_{z}-\alpha_{z} C_{x}+\alpha_{x} \beta_{z}-\alpha_{z} \beta_{x}\right) \\
\dot{z} & =f_{3}(x, y, z)+\mu\left(H_{x} \beta_{y}-H_{y} \beta_{x}+\alpha_{x} C_{y}-\alpha_{y} C_{x}+\alpha_{x} \beta_{y}-\alpha_{y} \beta_{x}\right)
\end{array},\right.
$$

where $\alpha, \beta$ are arbitrary differentiable functions on $\Omega$.

We apply this procedure to the $T$ system [21] given by

$$
\left\{\begin{array}{l}
\dot{x}=-a x+a y \\
\dot{y}=(c-a) x-a x z \quad, a, b, c \in \mathbb{R}, a \neq 0 . \\
\dot{z}=-b z+x y
\end{array}\right.
$$

A Hamilton-Poisson part of system (2) is given by

$$
\left\{\begin{array}{l}
\dot{x}=a y \\
\dot{y}=-a x z \quad, a, b, c \in \mathbb{R}, a \neq 0 . \\
\dot{z}=x y
\end{array}\right.
$$

Indeed, considering the functions $H, C \in C^{\infty}\left(\mathbb{R}^{3}, \mathbb{R}\right)$ given by

$$
H=\frac{1}{2} x^{2}-a z, \quad C=\frac{1}{2} y^{2}+\frac{a}{2} z^{2}
$$

by (3) we have $\frac{d H}{d t}=x \dot{x}-a \dot{z}=0$ and $\frac{d C}{d t}=y \dot{y}+a z \dot{z}=0$, thus $H$ and $C$ are constants of motion. Moreover, the Jacobian matrix of the functions $H, C$ has the rank two on the set $\left\{(x, y, z) \in \mathbb{R}^{3}: y \neq 0\right\}$. Therefore the functions $H$ and $C$ are functionally independent. Furthermore, system (3) reads $\dot{\mathbf{x}}=\nabla H \times \nabla C$, thus $\mu=1$. Therefore, we obtain the following result.

Proposition 2.1 Let $\alpha, \beta$ be arbitrary differentiable functions on $\mathbb{R}^{3}$. Then a family of integrable deformations of system (2) is given by the following system

$$
\left\{\begin{array}{l}
\dot{x}=-a x+a y+a \beta_{y}+a z \alpha_{y}-y \alpha_{z}+\alpha_{y} \beta_{z}-\alpha_{z} \beta_{y} \\
\dot{y}=(c-a) x-a x z-x \beta_{z}-a \beta_{x}-a z \alpha_{x}-\alpha_{x} \beta_{z}+\alpha_{z} \beta_{x} \\
\dot{z}=-b z+x y+x \beta_{y}+y \alpha_{x}+\alpha_{x} \beta_{y}-\alpha_{y} \beta_{x}
\end{array}\right.
$$

$a, b, c \in \mathbb{R}$, where we denote $f_{x}:=\frac{\partial f}{\partial x}$.

It is easy to see if the deformation functions $\alpha$ and $\beta$ vanish, then system (5) becomes (2).

Notice that the functions added in right side of the $T$ system for obtaining system (5) can be viewed as external control inputs, hence system (5) is a $T$ system with controls. 


\section{Stabilization of the $T$ system}

In this section we obtain some feedback linear controls such that the controlled $T$ system becomes stable.

Recall that system (2) possesses one or three equilibrium states. Moreover, if $a, b, c>$ $0, c>a$, and $2 a^{2}+b c-a c<0$, then all three equilibrium states are unstable and, in addition, the system (2) has a chaotic behavior [21] for some positive values of these parameters $(a=2.1, b=0.6, c=30)$. In the following consider that the parameters fulfill the above conditions.

Denote

$$
\begin{aligned}
& u_{1}(x, y, z)=a \beta_{y}+a z \alpha_{y}-y \alpha_{z}+\alpha_{y} \beta_{z}-\alpha_{z} \beta_{y}, \\
& u_{2}(x, y, z)=-x \beta_{z}-a \beta_{x}-a z \alpha_{x}-\alpha_{x} \beta_{z}+\alpha_{z} \beta_{x}, \\
& u_{3}(x, y, z)=x \beta_{y}+y \alpha_{x}+\alpha_{x} \beta_{y}-\alpha_{y} \beta_{x} .
\end{aligned}
$$

Then system (5) has the form

$$
\left\{\begin{array}{l}
\dot{x}=-a x+a y+u_{1}(x, y, z) \\
\dot{y}=(c-a) x-a x z+u_{2}(x, y, z) \\
\dot{z}=-b z+x y+u_{3}(x, y, z)
\end{array}\right.
$$

and the functions $u_{1}, u_{2}, u_{3}$ can be considered as external control inputs. Therefore, they can be used to stabilize the $T$ system.

Consider that these control functions are linear. Then $\alpha_{x}, \alpha_{y}, \alpha_{z}, \beta_{y}, \beta_{z}$ are constants and $\beta_{x}$ is linear. Hence $\alpha=k_{1} x+k_{2} y+k_{3} z, \beta=q x^{2}+p_{1} x+p_{2} y+p_{3} z$, where the coefficients are real numbers. Therefore, the control functions (6) becomes

$$
\begin{aligned}
& u_{1}(x, y, z)=k_{2} p_{3}-k_{3} p_{2}+a p_{2}-k_{3} y+a k_{2} z \\
& u_{2}(x, y, z)=-k_{1} p_{3}+k_{3} p_{1}-a p_{1}-p_{3} x-2 a q x+2 q k_{3} x-a k_{1} z \\
& u_{3}(x, y, z)=k_{1} p_{2}-k_{2} p_{1}+p_{2} x-2 q k_{2} x+k_{1} y
\end{aligned}
$$

Now, we impose the condition that $O(0,0,0)$ to be an equilibrium state of system (7), that is

$$
k_{2} p_{3}-k_{3} p_{2}+a p_{2}=0,-k_{1} p_{3}+k_{3} p_{1}-a p_{1}=0, k_{1} p_{2}-k_{2} p_{1}=0 .
$$

We obtain the following solutions: $\left(k_{2}=\frac{k_{1} p_{2}}{p_{1}}, p_{3}=\frac{-a p_{1}+k_{3} p_{1}}{k_{1}}\right),\left(k_{1}=0, k_{2}=0, k_{3}=a\right)$, $\left(k_{1}=0, p_{1}=0, p_{3}=\frac{-a p_{2}+k_{3} p_{2}}{k_{2}}\right),\left(p_{1}=0, p_{2}=0, p_{3}=0\right)$, and $\left(k_{1}=0, k_{2}=0, p_{1}=0, p_{2}=0\right)$. Each of these solutions is a minimal condition for which $O(0,0,0)$ is an equilibrium point of the considered $T$ system with controls.

\subsection{Stabilization of the $T$ system via a linear control about $O x$ axis}

In this case, we consider that $u_{2}=u_{3}=0$, which implies

$$
-p_{3}-2 a q+2 q k_{3}=0, k_{1}=0, p_{2}-2 q k_{2}=0 .
$$

Together with conditions (9), these relations lead to the following situations:

(i) $k_{1}=0, k_{2}=0, k_{3}=k, p_{1}=0, p_{2}=0, p_{3}=2 q(k-a)$, where $k \in \mathbb{R}$, thus $u_{1}=-k y$;

(ii) $k_{1}=0, k_{2}=0, k_{3}=a, p_{1}=p, p_{2}=0, p_{3}=0$, where $p \in \mathbb{R}$, thus $u_{1}=-a y$;

(iii) $k_{1}=0, p_{1}=0, k_{2}=r, k_{3}=k, p_{2}=2 q r, p_{3}=2 q(k-a)$, where $k, r \in \mathbb{R}$, thus $u_{1}=$ $-k y+a r z$. 
In the case (ii), system (7) has a family of equilibrium states, namely $(0, M, 0), M \in \mathbb{R}$. We do not consider this case.

Let us analyze the other cases.

(i) In this case, system (7) becomes

$$
\left\{\begin{array}{l}
\dot{x}=-a x+a y-k y \\
\dot{y}=(c-a) x-a x z \\
\dot{z}=-b z+x y
\end{array} .\right.
$$

The above system is the $T$ system with the parametric control $u_{1}(x, y, z)=-k y$, where $k$ is the tunning parameter (see, e.g. [3]).

Proposition 3.1 Let $a, b, c, k \in \mathbb{R}$ such that $a, b, c>0, c>a, 2 a^{2}+b c-a c<0$, and $k>a$. Then system (10) has an unique equilibrium state, namely $O(0,0,0)$, and it is asymptotically stable. More precisely, $O$ is a node if $k \leq \frac{4 a c-3 a^{2}}{4(c-a)}$, and a focus-node otherwise.

Proof. The equilibrium states of system (10) satisfy the relations

$$
-a x+a y-k y=0,(c-a) x-a x z=0,-b z+x y=0 .
$$

Then $x=0$ implies $y=z=0$, thus $O(0,0,0)$ is an equilibrium state. If $x \neq 0$, then we obtain $x=\frac{a-k}{a} y, z=\frac{c-a}{a}$, and $(a-k) y^{2}=b(c-a)$, which is false. Hence system (10) has not other equilibrium states.

Let $J$ be the Jacobian matrix of system (10), that is

$$
J(x, y, z)=\left[\begin{array}{ccc}
-a & a-k & 0 \\
c-a-a z & 0 & -a x \\
y & x & -b
\end{array}\right] .
$$

Then the eigenvalues of $J(0,0,0)$ are

$$
\lambda_{1}=-b, \lambda_{2,3}=\frac{-a \pm \sqrt{-3 a^{2}+4 a c+4 a k-4 c k}}{2} .
$$

If $k \leq \frac{4 a c-3 a^{2}}{4(c-a)}$, then all the eigenvalues are negative real numbers and, consequently, $O$ is an asymptotically stable node. If $k>\frac{4 a c-3 a^{2}}{4(c-a)}$, then $\lambda_{1}<0$ and $\lambda_{2,3}$ are conjugate complex numbers with negative real part. Therefore $O$ is an asymptotically stable focus-node, which finishes the proof.

For some values of the parameters, the chaotic behavior and the stabilized trajectories are shown in Figures 1-3.

(iii) In this case we have obtained a similar result, which generalizes the above result. System (7) becomes

$$
\left\{\begin{array}{l}
\dot{x}=-a x+a y-k y+a r z \\
\dot{y}=(c-a) x-a x z \\
\dot{z}=-b z+x y
\end{array}\right.
$$

Proposition 3.2 Let $a, b, c, k, r \in \mathbb{R}$ such that $a, b, c>0, c>a, 2 a^{2}+b c-a c<0, k>a$, and $-\sqrt{\frac{4 b(k-a)}{c-a}}<r<\sqrt{\frac{4 b(k-a)}{c-a}}$. Then system (11) has an unique equilibrium state, namely $O(0,0,0)$, and it is asymptotically stable. More precisely, $O$ is a stable node if $k \leq \frac{4 a c-3 a^{2}}{4(c-a)}$, and a stable focus-node otherwise. 


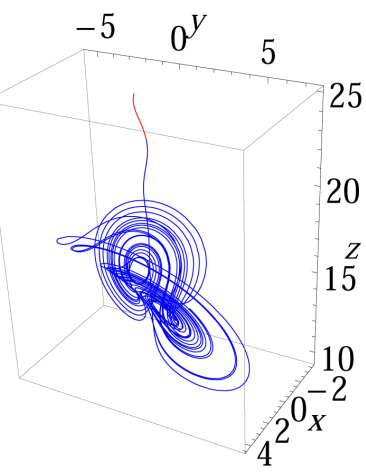

Figure 1. The chaotic attractor: an orbit of the $T$ system corresponding to the initial conditions $(0.01,0.01,14.01)$ and parameters $a=2, b=0.2, c=30$.

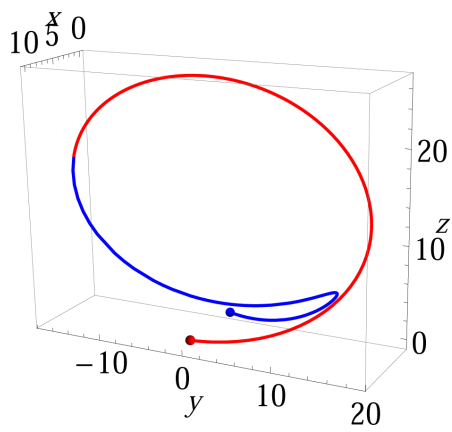

Figure 2. A stabilized trajectory corresponding to the initial conditions $(10,0.6,0.5)$ (the red disk) and parameters $a=2, b=0.2, c=30, k=2.013 ; O(0,0,0)$ is a stable node(the blue disk).

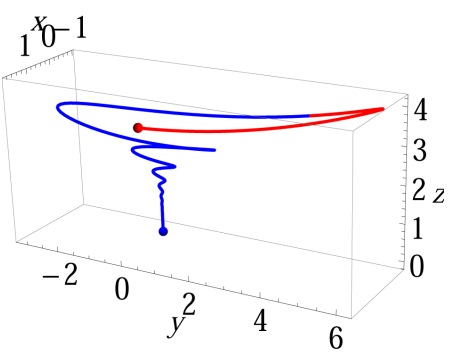

Figure 3. A stabilized trajectory corresponding to the initial conditions $(1.8,0.6,3.5)$ (the red disk) and parameters $a=2, b=0.2, c=30, k=3 ; O(0,0,0)$ is a stable focus-node (the blue disk).

\subsection{Stabilization of the $T$ system via linear controls about $O x$ and $O y$ axes}

Consider $u_{3}=0$. By (6) it follows $k_{1}=0, k_{2} p_{1}=0, p_{2}=2 q k_{2}$. Using (9), we obtain:

(i) $k_{1}=0, k_{2}=0, k_{3}=k, p_{1}=0, p_{2}=0, p_{3}=p$, where $k, p \in \mathbb{R}$, thus $u_{1}=-k y$ and $u_{2}=(-p-2 a q+2 k q) x$

(ii) $k_{1}=0, k_{2}=0, k_{3}=a, p_{1}=l, p_{2}=0, p_{3}=p$, where $l, p \in \mathbb{R}$, thus $u_{1}=-a y$ and 
$u_{2}=-p x$

(iii) $k_{1}=0, p_{1}=0, k_{2}=r, k_{3}=k, p_{2}=2 q r, p_{3}=2 q(k-a)$, where $k, r \in \mathbb{R}$, thus $u_{1}=-k y+a r z$ and $u_{2}=0$. This case was already studied.

In the case (ii), system (7) has a family of equilibrium states, namely $(0, M, 0), M \in \mathbb{R}$, which do not correspond to our purpose. It only remains the case (i), namely

$$
\left\{\begin{array}{l}
\dot{x}=-a x+a y-k y \\
\dot{y}=(c-a) x-(p+2 a q-2 k q) x-a x z \\
\dot{z}=-b z+x y
\end{array} .\right.
$$

As in previous subsection, we obtain the following result.

Proposition 3.3 Let $a, b, c, k, p \in \mathbb{R}$ such that $a, b, c>0, c>a, 2 a^{2}+b c-a c<0$. If $k<a, p>-a+c-2 a q+2 k q$ or $k>a, p<-a+c-2 a q+2 k q$, then system (12) has an unique equilibrium state, namely $O(0,0,0)$, and it is asymptotically stable.

In addition, if $-3 a^{2}+4 a c+4 a k-4 c k-4 a p+4 k p-8 a^{2} q+16 a k q-8 k^{2} q \geq 0$, then $O$ is a node, otherwise it is a focus-node.

Notice that if $p=q=0$, then Proposition 3.3 becomes Proposition 3.1.

\subsection{Stabilization of the $T$ system via three linear controls}

A more general case is obtained if all control functions are nonzero. In order to obtain simpler control functions and few new parameters, we choose the deformation functions $\alpha=k x+a z$ and $\beta=0$. We obtain $u_{1}=-a y, u_{2}=-a k z, u_{3}=k y$. Then system (5) becomes

$$
\left\{\begin{array}{l}
\dot{x}=-a x \\
\dot{y}=(c-a) x-a x z-a k z \quad, a, b, c, k \in \mathbb{R}, a, k \neq 0 . \\
\dot{z}=-b z+x y+k y
\end{array}\right.
$$

Again, under some conditions, the $T$ system is stabilized by the considered system with controls. More precisely, we have the next result.

Proposition 3.4 Let $a, b, c>0$ and $k \in \mathbb{R}, k \neq 0$. Then system (13) has an unique equilibrium point, namely $O(0,0,0)$ and it is asymptotically stable. Moreover, if $-\frac{b}{2 \sqrt{a}} \leq k \leq \frac{b}{2 \sqrt{a}}$, then $O$ is a node, otherwise it is a focus-node.

Remark 3.1 Consider the motion of a system $\dot{\mathbf{x}}=\mathbf{f}(\mathbf{x}), \mathbf{x}=(x, y, z), \mathbf{f}=\left(f_{1}, f_{2}, f_{3}\right)$ in the phase space as the flow of the fluid. Hence the logarithmic rate of the volume change is expressed as $\frac{1}{V} \frac{d V}{d t}=\nabla \cdot f[16]$, where the divergence of the vector field $\mathbf{f} i s \nabla \cdot f=\frac{\partial f_{1}}{\partial x}+\frac{\partial f_{2}}{\partial y}+\frac{\partial f_{3}}{\partial z}$. If the divergence is less than zero, then the system is called dissipative and it have "a state space volume that decreases on average along the trajectory so that the orbit approaches an attractor of measure zero in the state space" [20].

In the case of the $T$ system, the divergence is $\nabla \cdot \mathbf{f}=-a-b<0$ (in the considered case $a, b>0$ ). It is easy to see that in all considered controlled system in this paper the divergence is also $-a-b$. On the other hand, the control functions used in other papers change the divergence of the system (see, e.g., [5, 22]).

Acknowledgemens. This research was partially supported by Horizon2020-2017-RISE777911 project. 


\section{References}

[1] A. Babloyantz, C. Nicolis, and J. M. Salazar, Physics Letters A 111, 152-156 (1985).

[2] S. Boccaletti, C. Grebogi, Y.-C. Lai, H. Mancini, and D. Maza, Physics Reports 329, 103-197 (2000).

[3] G. Chen, and X. Dong, From Chaos to Order: Methodologies, Perspectives and Applications (World Scientific Pub. Co., Singapore, 1998) 753 pp.

[4] G. Chen, Controlling Chaos and Bifurcations in Engineering Systems (CRC Press, Boca Raton, FL, 1999) 688 pp.

[5] Y. Chen, and Z.-Y. Yan, Communications in Theoretical Physics 49(4), 951-954 (2008).

[6] R. Constantinescu, C. Ionescu, Physics AUC 21-Sp.Issue, 207-215 (2011).

[7] A. Galajinsky, Journal of Mathematical Analysis and Applications 416(2), 995-997 (2014).

[8] C. Lăzureanu, Comptes Rendus Mathematique 355(5), 596-600 (2017).

[9] C. Lăzureanu, Advances in Mathematical Physics 2017, Article ID 4596951, 9 pages (2017).

[10] C. Lăzureanu, International Journal of Bifurcation and Chaos in Applied Sciences and Engineering 28(5), Article ID 1850066, 7 pages (2018).

[11] C. Lăzureanu, C. Hedrea, and C. Petrişor, International Conference on Applied Mathematics \& Computer Science (ICAMCS), Paris, France, 2018, pp. 1-18.

[12] C. Lăzureanu, and C. Petrişor, Advances in Mathematical Physics 2018, Article ID 5398768, 9 pages (2018).

[13] C. Lăzureanu, AIP Conference Proceedings 2116, 370004 (2019); https://doi.org/10.1063/1.5114377.

[14] C. Lăzureanu, C. Hedrea, and C. Petrişor, ICCMAE 2018, ITM Web Conf. 2901015 (2019); https://doi.org/10.1051/itmconf/20192901015.

[15] C. Lăzureanu, and C. Căplescu, Proceedings of The XV-th Int. Conf. on Mathematics and its Applications, Timişoara, 2019, 90-97.

[16] E. N. Lorenz, Journal of the Atmospheric Sciences 20(2), 130-141 (1963).

[17] J. Lü, G. Chen, and S. Zhang, International Journal of Bifurcation and Chaos 12(5), 1001-1015 (2002).

[18] L. M. Pecora, and T. L. Carroll, Physical Review A 44(4), 2374-2383 (1991).

[19] M. I. Rabinovich, and H. D. I. Abarbanel, Neuroscience 87, 5-14 (1998).

[20] J. C. Sprott, Physics Letters A 378, 1361-1363 (2014).

[21] Gh. Tigan, Sci. Bull. Politehnica University of Timisoara 50(64), Fascicola 1, 61-72 (2005).

[22] Gh. Tigan, Carpathian Journal of Mathematics 22(1-2), 153-161 (2006).

[23] G. Tigan, and D. Opris, Chaos, Solitons \& Fractals 36, 1315-1319 (2008).

[24] T. Yang, and L. O. Chua, IEEE Transactions on Circuits and Systems I: Fundamental Theory and Applications 44(10), 976-988 (1997). 